\title{
Poly(3,4-ethylenedioxythiophene)-reduced graphene oxide/copper-based metal-organic frameworks as a high-performance symmetrical supercapacitor device
}

Dharshini Mohanadas

Universiti Putra Malaysia

Muhammad Amirul Aizat Mohd Abdah

Universiti Putra Malaysia

Nur Hawa Nabilah Azman

Universiti Putra Malaysia

Thahira B.S.A. Ravoof

Universiti Putra Malaysia

Yusran Sulaiman ( $\nabla$ yusran@upm.edu.my )

Universiti Putra Malaysia

\section{Research Article}

Keywords: PrGO/HKUST-1, PrGO, PEDOT, 3,4-ethylenedioxythiophene, KCl/PVA

Posted Date: January 14th, 2021

DOI: https://doi.org/10.21203/rs.3.rs-141960/v1

License: (c) (i) This work is licensed under a Creative Commons Attribution 4.0 International License.

Read Full License 


\title{
Poly(3,4-ethylenedioxythiophene)-reduced graphene oxide/copper-based metal-organic frameworks as a high-performance symmetrical supercapacitor device
}

Dharshini Mohanadas ${ }^{1}$, Muhammad Amirul Aizat Mohd Abdah¹, Nur Hawa Nabilah Azman ${ }^{3}$, Thahira

B.S.A. Ravoof ${ }^{1,2}$ and Yusran Sulaiman ${ }^{1,3^{*}}$

${ }^{1}$ Department of Chemistry, Faculty of Science, Universiti Putra Malaysia, 43400 UPM Serdang, Selangor, Malaysia.

${ }^{2}$ Foundry of Reticular Materials for Sustainability (FORMS), Materials Synthesis and Characterization Laboratory, Institute of Advanced Technology, Universiti Putra Malaysia, 43400 Serdang, Selangor, Malaysia.

${ }^{3}$ Fuctional Devices Laboratory, Institute of Advanced Technology (ITMA), Universiti Putra Malaysia, 43400 UPM, Serdang, Selangor, Malaysia.

*Corresponding author (yusran@upm.edu.my)

\begin{abstract}
A novel poly(3,4-ethylenedioxythiophene)-reduced graphene oxide/copper-based metal-organic framework (PrGO/HKUST-1) has been successfully fabricated by incorporating electrochemically synthesized poly $(3,4-$ ethylenedioxythiophene)-reduced graphene oxide (PrGO) and hydrothermally synthesized copper-based metalorganic framework (HKUST-1). The field emission scanning microscopy (FESEM) and elemental mapping analysis revealed an even distribution of poly(3,4-ethylenedioxythiophene) (PEDOT), reduced graphene oxide (rGO) and HKUST-1. The crystalline structure and vibration modes of PrGO/HKUST-1 was validated utilizing X-ray diffraction (XRD) as well as Raman spectroscopy, respectively. A remarkable specific capacitance $(360.5 \mathrm{~F} / \mathrm{g})$ was obtained for PrGO/HKUST-1 compared to HKUST-1 (103.1 F/g), PrGO (98.5 F/g) and PEDOT (50.8 F/g) using KCI/PVA as a gel electrolyte. Moreover, PrGO/HKUST-1 composite with the longest charge/discharge time displayed excellent specific energy $(21.0 \mathrm{Wh} / \mathrm{kg})$, specific power $(479.7 \mathrm{~W} / \mathrm{kg})$ and outstanding cycle life $(95.5 \%)$ over 4000 cycles. Thus, the PrGO/HKUST-1 can be recognized as a promising energy storage material.
\end{abstract}

\section{Introduction}

Supercapacitors are electrical devices which have an excellent energy storage system compared to conventional capacitors as it possesses high specific power, long-term cycling life as well as rapid charging/discharging time ${ }^{1-4}$. Supercapacitors comprise four major components which are current collectors, active materials, an electrolyte and a separator ${ }^{5}$. Supercapacitors can be categorized as pseudocapacitors and electric double layer capacitors (EDLCs) based on their charge storage mechanism.

Faradaic redox reactions occur at an electroactive material surface in order to store charge in pseudocapacitors. In contrast, the charges are stored electrostatically in EDLCs, where adsorption/desorption of ion occurs at the electrode/electrolyte interface. Mainly, conducting polymers (CPs) and transition metal oxides (TMOs) are classified as pseudocapacitor materials while EDLCs are derived from carbon-based materials, for instance, graphene oxide (GO), multi-walled carbon nanotube (MWCNT), reduced graphene oxide (rGO) and activated carbon. Pseudocapacitors exhibit relatively high specific capacitance than EDLCs, however, it shows low specific power and short cycling life. In comparison to pseudocapacitors, EDLCs show higher specific power, excellent life cycle but lower specific energy. Thus, the combination of pseudocapacitors and EDLCs as hybrid supercapacitors could enhance the supercapacitive performance by exhibiting excellent specific capacitance, superior cyclability, enormous specific power along with satisfying specific energy.

Poly(3,4-ethylenedioxythiophene) (PEDOT), polyaniline (PANI), polypyrrole (PPy), polythiophene (PTh) and its derivatives are promising conducting polymer candidates for supercapacitor applications. However, PEDOT has received more attention among CPs candidates because of its outstanding properties i.e. good electrical conductivity, wide operating potential, environmentally friendly ${ }^{6}$ and excellent stability at its oxidized state ${ }^{1,7}$. Over 
the last few years, metal-organic frameworks (MOFs) have received great attention and are labeled as the most promising electrochemical candidate in supercapacitor applications due to their high internal pore volume ${ }^{8}$ and high surface area ${ }^{9}$. MOFs are crystalline porous solids, which consist of metal ions as well as organic linkers that are held together by strong covalent bonds ${ }^{4,10}$. HKUST-1 is a copper-based MOF that composed of copper ion (metal ion) as well as trimesic acid (organic ligand) ${ }^{11,12}$. HKUST-1 is being explored in supercapacitor applications because it exhibits high surface area ${ }^{13}$, superior pore volume ${ }^{14}$, and high thermal stability ${ }^{1}$. However, HKUST-1 suffers from poor electrical conductivity. CPs and MOFs are usually incorporated with carbon-based materials to enhance their properties by providing high mechanical strength and electrical conductivity ${ }^{15}$. One of the carbonbased materials which can boost the stability of CP/MOF composite is rGO. Zhu et al., ${ }^{16}$ reported $p$-toluenesulfonic doped PPy/rGO composite possesses an excellent specific capacitance $(280.3 \mathrm{~F} / \mathrm{g})$ and the presence of rGO successfully enhanced the stability of PPy with $92 \%$ energy retention even after 10000 cycles. A zinc-based MOF (MOF-5)/rGO composite was prepared by Wen et al., ${ }^{17}$ via a solvothermal followed by the annealing process. The composite displayed an enhanced specific capacitance $(312 \mathrm{~F} / \mathrm{g}$ ) and $81 \%$ retention of capacitance over 5000 cycles. A solid-type symmetrical HKUST-1/rGO deposited on a carbon fiber paper also revealed an excellent charge storage capacity $(198 \mathrm{~F} / \mathrm{g})$ along with excellent cyclability ${ }^{18}$.

Here, we successfully fabricated a novel composite consisting of poly(3,4-ethylenedioxythiophene)-reduced graphene oxide/copper-based metal-organic framework (PrGO/HKUST-1) with excellent performance for energy storage. The PrGO was electrochemically prepared while HKUST-1 was synthesized hydrothermally and it was casted on top of the PrGO layer. The synthesized PrGO/HKUST-1 was then characterized by FESEM, elemental mapping, XRD, Raman spectroscopy as well as electrochemical measurements. PEDOT was chosen because of its good electrical conductivity while HKUST-1 possesses a high surface area. A synergistic effect between PEDOT, rGO and HKUST-1 leads to an excellent specific capacitance, specific energy, high specific power and superior cycling life.

\section{Results and Discussion}

FESEM was performed to identify the surface morphology of the materials as depicted in Figure 1(a). PEDOT (Figure 1(a)(i)) reveals a homogeneous granular morphology similar to the typical polymeric structure. PrGO displays a wrinkle-like sheet morphology (Figure 1(a)(ii)), which is contributed by the rGO. This morphology demonstrates the successful incorporation of PEDOT and rGO. This wrinkled-like sheet morphology provides a high surface area which enables efficient ion diffusion. The HKUST-1 (Figure 1(a)(iii)) displays a typical octahedral shape morphology, whereas the PrGO/HKUST-1 demonstrates the presence of HKUST-1 and PrGO as both the octahedral morphology as well as wrinkle-like rGO sheet covered with PEDOT grains are observed in Figure 1 (a)(iv).

The PrGO/HKUST-1 (Figure 1(b)(i)) was further analyzed via elemental mapping (Figure 1(b)(ii-v)) to study the elements that exist in the composite. Carbon (C), oxygen (O), sulfur (S) and copper (Cu) are evenly distributed on the PrGO/HKUST-1 surface, indicating the homogeneous formation of the composite. S and Cu elements signify the distribution of PEDOT and HKUST-1, respectively ${ }^{19,20}$. The presence of $\mathrm{C}$ and $\mathrm{O}$ elements are originated from PEDOT, rGO and HKUST-1. 


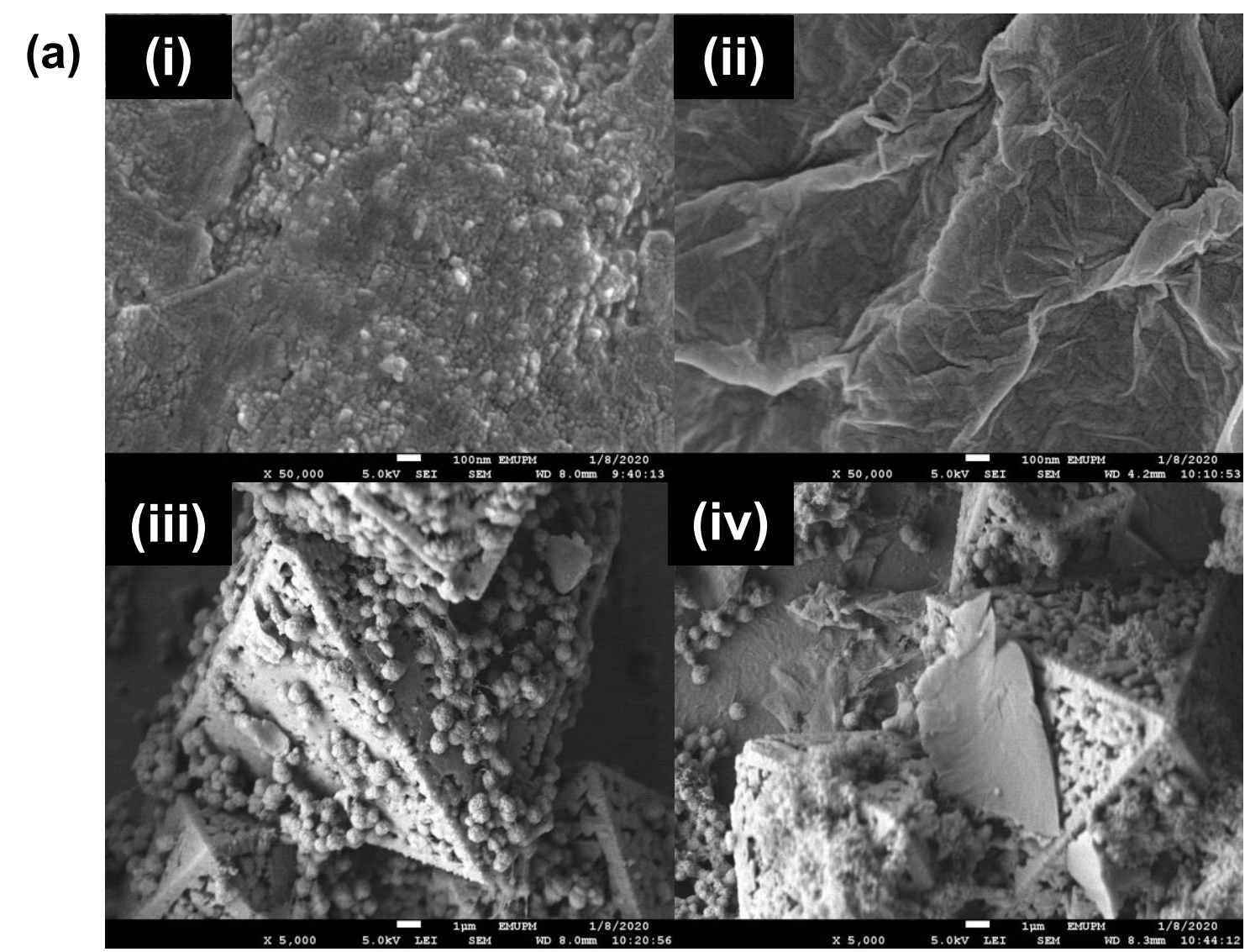

(b)
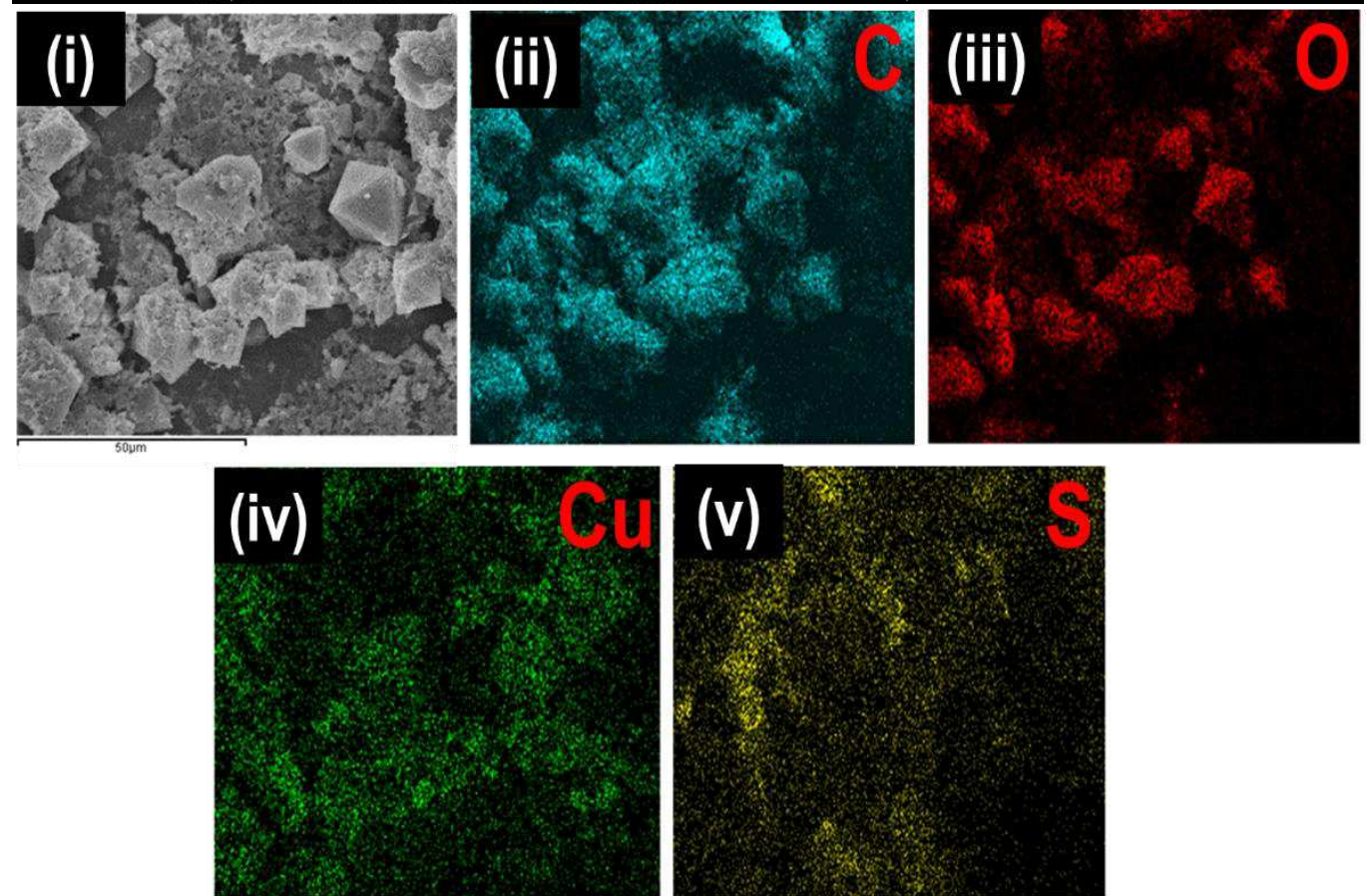

Figure 1: (a) FESEM images of (i) PEDOT (scale bar: $100 \mathrm{~nm}$ ), (ii) PrGO (scale bar: $100 \mathrm{~nm}$ ), (iii) HKUST-1 (scale bar: $1 \mu \mathrm{m}$ ), (iv) PrGO/HKUST-1 (scale bar: $1 \mu \mathrm{m}$ ) and (b) elemental mapping of (i) PrGO/HKUST-1 (scale bar: 50 $\mu \mathrm{m}$ ) with (ii) $\mathrm{C}$, (iii) $\mathrm{O}$, (iv) $\mathrm{Cu}$ and (v) S distributions.

The crystallinity of the composites was evaluated using XRD (Figure 2). A diffraction peak at $2 \theta=25.8^{\circ}(020)$ indicates the presence of interchain planar ring stacking of PEDOT (Figure 2(a)) ${ }^{21,22}$. PrGO (Figure 2(b)) composite reveals a diffraction peak $\left(2 \theta=25.6^{\circ}\right)$, representing the $(020)$ and $(002)$ lattice planes of PEDOT and $\mathrm{rGO}$, respectively. The XRD peak of $\mathrm{PrGO}$ only shows one diffraction peak $\left(2 \theta=25.6^{\circ}\right)$ as the peak of $\mathrm{rGO}$ is overlapping with PEDOT. The disorder in the rGO sheets appears when the majority of oxygenated functional groups were successfully reduced from the GO sheet during the electrodeposition method ${ }^{23}$. The PrGO composite does not 
show any diffraction peak at $2 \theta=10^{\circ}$ (peak for GO), which further verifies the reduction of GO to rGO ${ }^{24}$. The assynthesized HKUST-1 (Figure 2(d)) displays similar diffraction peaks as the simulated HKUST-1 (Figure 2(c)) at $6.7^{\circ}(200), 9.5^{\circ}(220), 11.6^{\circ}(222), 13.5^{\circ}(400), 17.5^{\circ}(400), 19.1^{\circ}(600), 26.0^{\circ}(731)$ and $29.4^{\circ}(751)$, revealing a successful synthesis of HKUST-1 via hydrothermal method ${ }^{25,26}$. All the XRD diffraction peaks of PEDOT, rGO and HKUST-1 are well-presented in PrGO/HKUST-1 (Figure 2(e)) spectrum. The results demonstrate that the framework of HKUST-1 is retained during the synthesis process which is well supported by FESEM images ((Figure 1(a)(iii) and Figure 1(a)(iv)).

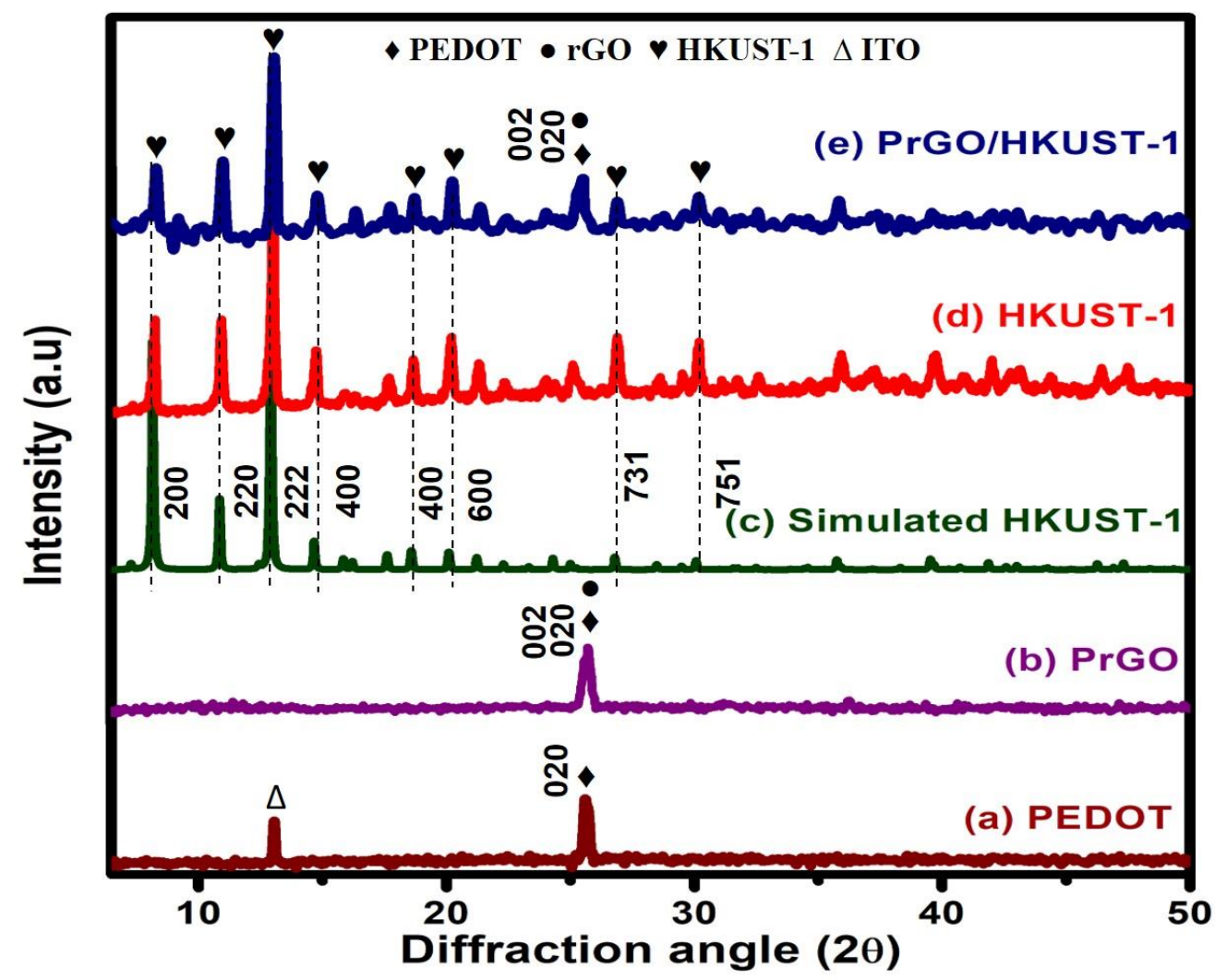

Figure 2: XRD patterns of (a) PEDOT, (b) PrGO, (c) simulated HKUST-1, (d) HKUST-1 and (e )PrGO/HKUST-1.

The vibrational modes of the different materials were examined via Raman spectroscopy (Figure 3). The PEDOT (Figure 3(a)) displays peaks at 440, 576, and $989 \mathrm{~cm}^{-1}$, corresponding to the oxyethylene ring of EDOT monomer deformation. The C-O-C mode can be observed at $1107 \mathrm{~cm}^{-1}$ while $\mathrm{C}_{\beta}-\mathrm{C}_{\beta}, \mathrm{C}_{\alpha}-\mathrm{C}_{\alpha}$, symmetry and asymmetry $\mathrm{C}=\mathrm{C}$ stretching modes of PEDOT are seen at 1260,1366, 1427 and $1526 \mathrm{~cm}^{-1}$, respectively ${ }^{7}$. The combination of PEDOT and rGO (Figure 3(b)) displays two obvious peaks at 1356 and $1600 \mathrm{~cm}^{-1}$, resembling the D band ( $s p^{3}$ hybridized carbon) and $G$ band ( $s p^{2}$-hybridized carbon) where the intensity ratio of $D$ and $G$ band (ID/IG) represents the degree of disorder in graphitic material ${ }^{27}$. ID/lG ratio more than 1 indicates the presence of high $s p^{3}$-hybridized carbon atoms compared to $s p^{2}$-hybridized carbon ${ }^{28}$. The calculated ID/lG ratio of PrGO is 1.24 , which shows a high degree of disorder in PrGO, revealing the majority of oxygenated functional groups in GO have been reduced successfully 29 . HKUST-1 (Figure 3(c)) displays all vibration modes of $\mathrm{Cu}$ (II) species at low frequency region (150 to $\left.600 \mathrm{~cm}^{-1}\right)$. The Raman peak at $177 \mathrm{~cm}^{-1}$ exhibits the presence of $\mathrm{Cu}-\mathrm{Cu}$ dimer stretching mode, while the $\mathrm{Cu}-\mathrm{O}$ vibration mode of HKUST-1 can be detected at 278 and $501 \mathrm{~cm}^{-1}$, respectively. The C-H out-of-plane ring bending modes of trimesic acid are detected at 744 and $827 \mathrm{~cm}^{-1}$ whereas the $\mathrm{C}=\mathrm{C}$ stretching mode of the trimesic acid benzene ring is spotted at $1006 \mathrm{~cm}^{-1}$. Raman peaks of HKUST-1 (Figure 3(c)) observed at 1457 and $1544 \mathrm{~cm}^{-1}$ indicate asymmetry and symmetry C-O stretching modes, respectively. HKUST-1 incorporated with PrGO still exhibits all Raman peaks of PEDOT, rGO and HKUST-1, demonstrating the successful combination of PrGO/ HKUST-1 (Figure 3(d)). 


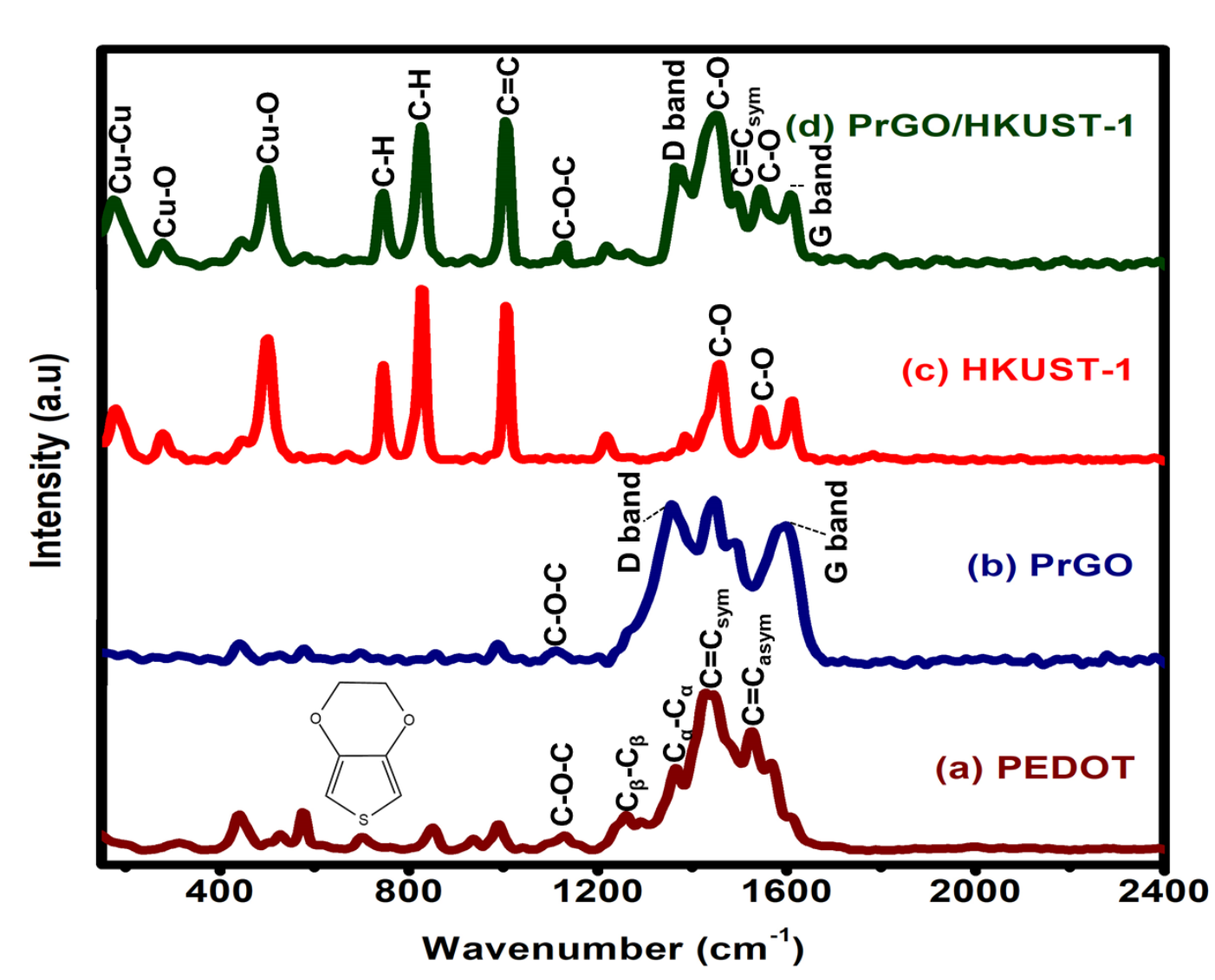

Figure 3: Raman spectra of (a) PEDOT, (b) PrGO, (c) HKUST-1 and (d) PrGO/HKUST-1

The electrochemical properties of the composites were evaluated in a three-electrode configuration. From Figure 4(a), PEDOT reveals a quasi-rectangular CV shape, suggesting the pseudocapacitance characteristic. PrGO displays a nearly rectangular CV curve, indicating EDLC characteristics. This result shows that rGO is dominant in the PrGO composite. CV curve of HKUST-1 displays a redox peak that confirms the faradic charge storage mechanism in HKUST-1. Interestingly, the integration of PrGO with HKUST-1 (PrGO/HKUST-1) has significantly increased the redox peak currents, where the peaks are mainly contributed by the pseudocapacitance characteristic of HKUST-1. The oxidation peak demonstrates the oxidation of $\mathrm{Cu}^{+}$to $\mathrm{Cu}^{2+}$ while the reduction peak shows the reduction of $\mathrm{Cu}^{2+}$ to $\mathrm{Cu}^{+30}$. The electrochemical reactions that occur in the HKUST-1 can be explained using equation (1) ${ }^{31}$ :

$\left(\mathrm{Cu}^{2+} \mathrm{R}\right) \mathrm{n}+\mathrm{nK}^{+}$(gel electrolyte $)+\mathrm{ne}^{-} \rightleftharpoons\left(\mathrm{Cu}^{+}\left(\mathrm{K}^{+}\right) \mathrm{R}\right) \mathrm{n}$

where $\mathrm{R}$ is the ligand of HKUST-1. When electrolyte cation $\left(\mathrm{K}^{+}\right)$enters the HKUST-1 network, HKUST-1 displays pseudocapacitive behavior as a reaction between copper ion $\left(\mathrm{Cu}^{2+/+}\right)$ and the electrolyte occurs, which mainly contributed from the $\mathrm{K}^{+}$ion insertion and deinsertion process ${ }^{31}$. Figure $4(\mathrm{~b})$ implies the CV curves of PrGO/HKUST1 at different scan rates ranging from 5 to $100 \mathrm{mV} / \mathrm{s}$. The redox current density of PrGO/HKUST-1 gradually intensifies as the scan rate increases. It can be clearly observed that PrGO/HKUST-1 is still able to maintain its CV shape with well-defined redox peaks without an evident distortion at a higher scan rate $(100 \mathrm{mV} / \mathrm{s})$, signifying a good rate capability of PrGO/HKUST-1 ${ }^{32,33}$.

The prepared materials were further analyzed via galvanostatic charge-discharge (GCD) analysis. Figure 4(c) illustrates the GCD curves of different materials at $1.8 \mathrm{~A} / \mathrm{g}$. PEDOT, HKUST-1 as well as PrGO/HKUST-1 demonstrate non-linear GCD curves ${ }^{34}$, indicating the good capacitive performance of materials with pseudocapacitive behavior 11,33,35 whereas PrGO exhibits nearly linear GCD curve, indicating EDLC behavior of the electrode ${ }^{18}$. PrGO/HKUST-1 depicts the longest discharging time compared to other individual samples with a small and negligible voltage drop (IR drop), demonstrating an outstanding specific capacitance as well as the low internal resistance of the electroactive material ${ }^{36}$. Figure $4(\mathrm{~d})$ presents GCD measurements of PrGO/HKUST-1 at different current density $(1.0-2.0 \mathrm{~A} / \mathrm{g})$. The GCD curves of PrGO/HKUST-1 clearly show the discharging time of the electrode reduces when the current density increases. This is because, at higher current density, the electrolyte ions movement is time limited, where only outer electroactive sites of the electrode are involved for the energy 
storage process. Moreover, the GCD curves retain non-linear GCD shapes at all current densities, demonstrating good electrochemical reversibility of PrGO/HKUST-1 ${ }^{37}$.
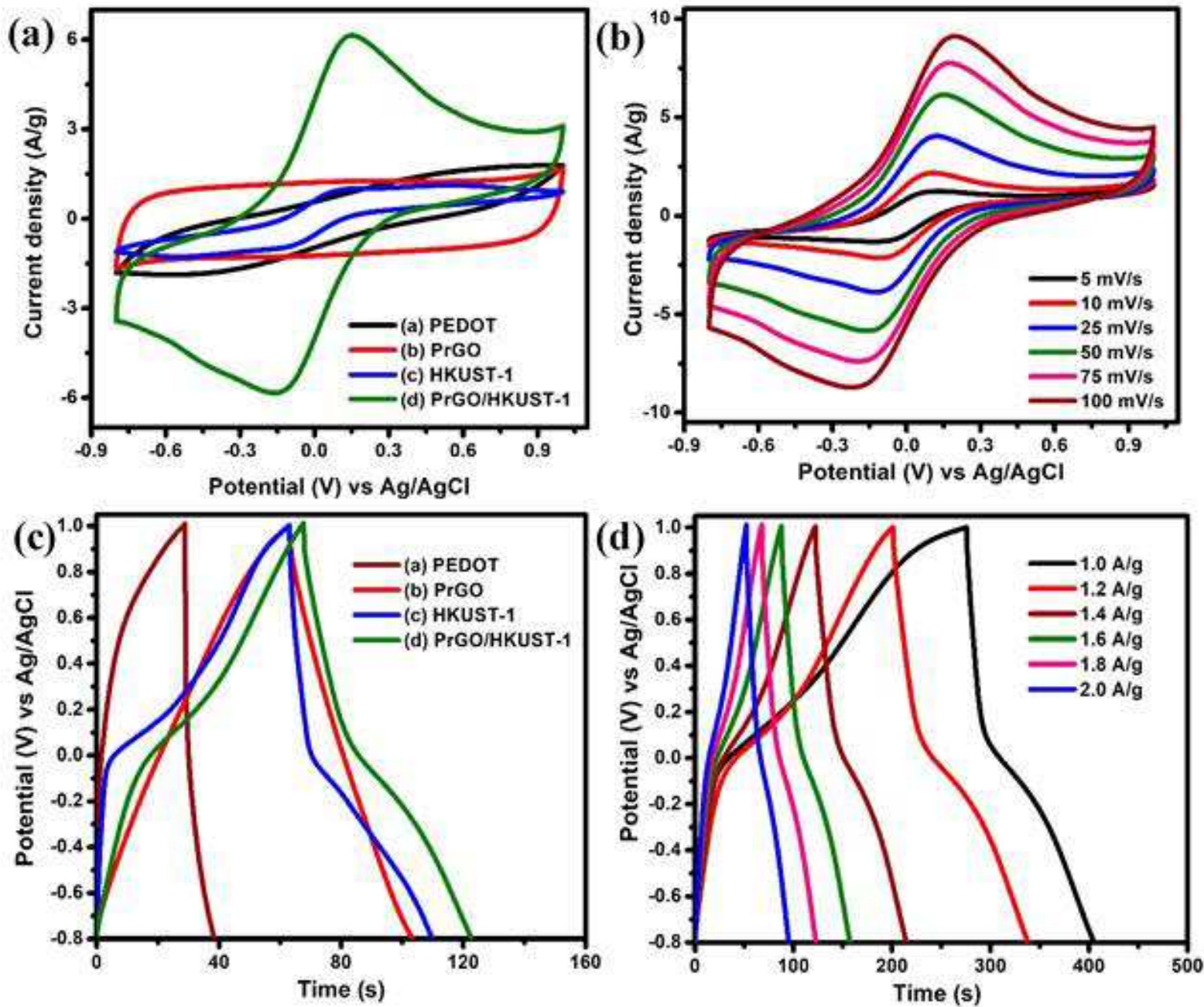

Figure 4: (a) CV curves of PEDOT, PrGO, HKUST-1 and PrGO/HKUST-1 at $50 \mathrm{mV} / \mathrm{s}$, (b) CV curves of PrGO/HKUST-1 at various scan rates (5-100 mV/s), (c) GCD analysis of PEDOT, PrGO, HKUST-1, PrGO/HKUST1 and (d) GCD plots of PrGO/HKUST-1 at various current density $(1.0-2.0 \mathrm{~A} / \mathrm{g})$.

The charge storage capacity of as-prepared symmetrical energy storage devices was examined via a two-electrode configuration using KCl/PVA gel as an electrolyte and separator ${ }^{1}$. Figure 5(a) depicts the CV curves of different materials at a potential range of 0 to $1 \mathrm{~V}$. Quasi rectangular CV shapes of PEDOT and HKUST-1 prove the pseudocapacitance behavior of PEDOT and HKUST-1, whereas PrGO displays a nearly rectangular CV curve, demonstrating EDLC characteristic. The PrGO/HKUST-1 depicts a quasi rectangular CV curve, suggesting a combination of EDLC and pseudocapacitance behavior ${ }^{38}$. PrGO/HKUST-1 reveals the largest CV curve, signifying the highest specific capacitance $\left(C_{\mathrm{sp}}\right)$ where the area under the $\mathrm{CV}$ curve indicates the quantity of electrical charge stored in an electrode ${ }^{13}$. $C_{\text {sp }}$ can be calculated using equation (2):

$C_{s p}=\frac{1}{2 m v \Delta V} \int I d V$

where $/ \mathrm{d} V$ indicates the integrated area of the CV curve while $m, v$ and $\Delta V$ exhibit mass of active material, potential scan rate (V/s) and potential window (CV) of sample, respectively. The $C_{\mathrm{sp}}$ obtained for PrGO/HKUST-1 is 360.5 $\mathrm{F} / \mathrm{g}$ where it is significantly greater compared to HKUST-1 (103.1 F/g), PrGO $(98.5 \mathrm{~F} / \mathrm{g})$ and PEDOT $(50.8 \mathrm{~F} / \mathrm{g})$ at a scan rate of $5 \mathrm{mV} / \mathrm{s}$. Figure $5(\mathrm{~b})$ implies the CV curves of symmetrical PrGO/HKUST-1 from the scan rate of 5 to $100 \mathrm{mV} / \mathrm{s}$. The current density and the area under the CV curves increase evidently as the scan rate is increased. The relationship between $C_{\mathrm{sp}}$ and scan rate is elucidated in Figure. 5(c) and the results confirm that $C_{\mathrm{sp}}$ reduces over the increasing scan rate. At slower scan rates, the electrolyte ions are able to utilize all the electroactive sites of the material and lead to a complete redox reaction which provides high $C_{\mathrm{sp}}{ }^{4}$. However, at faster scan rates, the movement of electrolyte ions is time limited which means that only the outer electroactive sites of the material are involved for energy storage, resulting in low $C_{\mathrm{sp}}{ }^{39}$. Interestingly, PrGO/HKUST-1 exhibits higher $C_{\mathrm{sp}}$ compared to other materials, proving incorporation of HKUST-1, PEDOT and rGO can successfully boost the electrochemical performance of PrGO/HKUST-1 composite, which mainly caused by the faradic redox reaction occur at the surface 
of electroactive material ${ }^{40}$. The $C_{\text {sp }}$ achieved in this work is higher in comparison to other reported HKUST-1 based supercapacitors i.e. reduced graphene oxide-metal organic framework (rGO/HKUST-1) (193.0 F/g) ${ }^{18}$ and copperbased metal-organic framework/polyaniline (HKUST-1/PANI) $(19.9 \mathrm{~F} / \mathrm{g}){ }^{11}$.

The as-prepared symmetrical devices were further evaluated via GCD measurements at a fixed current density $(1.8 \mathrm{~A} / \mathrm{g}$ ). The symmetrical PrGO/HKUST-1 (Figure 5(d)) device exhibits a non-linear GCD curve, revealing the presence of pseudocapacitive material that is dominant in the PrGO/HKUST-1. Furthermore, the symmetrical PrGO/HKUST-1 shows the longest discharging time, suggesting a high $C_{\mathrm{sp}}$ value. Figure $5(\mathrm{e})$ displays GCD measurements of symmetrical PrGO/HKUST-1 device at various current densities (1.0 to $2.0 \mathrm{~A} / \mathrm{g}$ ). The $C_{\mathrm{sp}}$ can be also obtained from GCD measurements utilizing equation (4), where the $I, \Delta \mathrm{t}, m$ and $\Delta \mathrm{V}$ refer to discharging current $(\mathrm{A})$, discharging time of the device (s), average mass of two electrodes (g) and cell operating potential (V), respectively.

$C_{\mathrm{sp}}=\frac{I \Delta t}{m \Delta V}$

The PrGO/HKUST-1 device exhibits a $C_{\mathrm{sp}}$ of $163.5 \mathrm{~F} / \mathrm{g}$ at $1.0 \mathrm{~A} / \mathrm{g}$, which declines to $104.2 \mathrm{~F} / \mathrm{g}$ at $2.0 \mathrm{~A} / \mathrm{g}$. The GCD results (Figure 5(e)) are in good agreement with the CV plots displayed in Figure 5(b). 

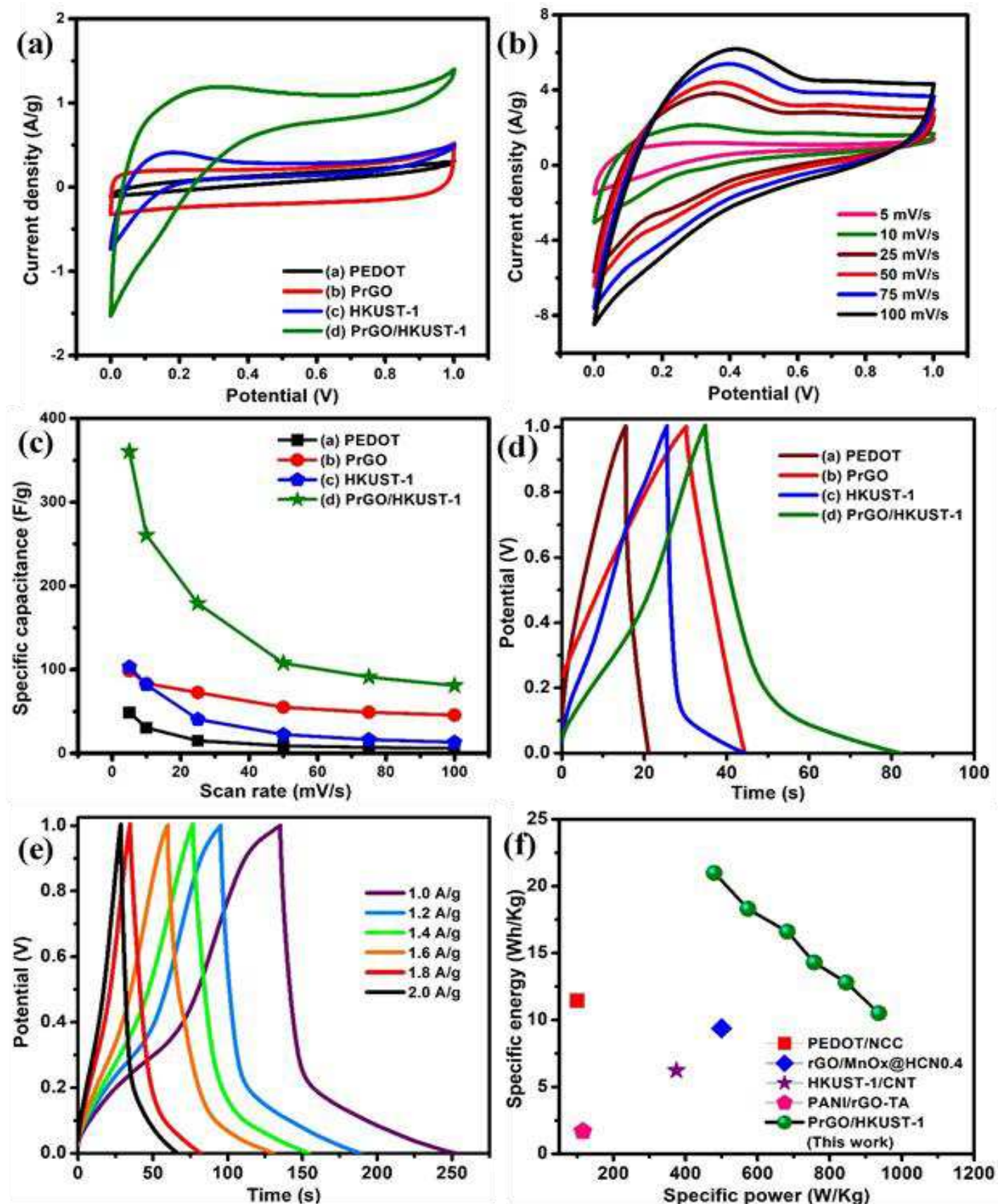

Figure 5: (a) CVs of PrGO, PEDOT, HKUST-1 and PrGO/HKUST-1 using KCI/PVA gel electrolyte at $5 \mathrm{mV} / \mathrm{s}$, (b) CVs of symmetrical PrGO/HKUST-1 at different scan rates $(5-100 \mathrm{mV} / \mathrm{s}$ ) and (c) graph of specific capacitance versus scan rate for PrGO, PEDOT, HKUST-1 and PrGO/HKUST-1, (d) GCD analysis of PEDOT, PrGO, HKUST1 and PrGO/HKUST-1 at a current density of $1.8 \mathrm{~A} / \mathrm{g}$ using KCl/PVA gel electrolyte, (e) GCD analysis of symmetrical PrGO/HKUST-1 at various current densities (1.0-2.0 A/g), (f) Ragone plots of PrGO/HKUST-1.

The specific energy $(E)$ and specific power $(P)$ of an electrode can be measured utilizing equations (4) and (5) where $C_{\mathrm{sp}}, \Delta \mathrm{V}, I$ and $m$ are the specific capacitance, potential window at discharging process, the current applied and mass of symmetrical electrode, respectively.

$$
\begin{aligned}
& E=\frac{C_{\mathrm{sp}} \Delta V^{2}}{2} \\
& P=\frac{\Delta V I}{2 m}
\end{aligned}
$$

Figure 5(f) reveals specific energy of $21 \mathrm{Wh} / \mathrm{kg}$ for PrGO/HKUST-1 at a specific power of $479.7 \mathrm{~W} / \mathrm{kg}$, where it is significantly greater compared to recently reported symmetrical supercapacitors; poly (3, 4ethylenedioxythipohene)/nanocrystalline cellulose (PEDOT/NCC) ${ }^{41}$, reduced graphene oxide/MnOx@carbon 
hollow nanospheres (rGO/MnO @ $\left.\mathrm{HCN}_{0.4}\right)^{42}$, polyaniline/reduced graphene oxide-tannic acid (PANI/rGO-TA) ${ }^{43}$ and copper-based metal-organic framework/carbon nanotube (HKUST-1/CNT) ${ }^{11}$.

The conductivity as well as the ion mobility at the interface of electrode/electrolyte were evaluated via EIS analysis by retrieving the information of the internal resistance along with the interface resistance amidst an electrode and electrolyte ${ }^{44}$. The Nyquist plots (Figure 6(a)) consist of equivalent series resistance (ESR) as well as the resistance of charge transfer $\left(R_{\mathrm{ct}}\right)$ at high-frequency region while the vertical line (Warburg line) at lowfrequency region. The ESR is the intersection point that appears at the real axis whereas $R_{\mathrm{ct}}$ is the semicircle diameter. The PrGO/HKUST-1 exhibits the lowest ESR (35.0 $\Omega$ ) and $R_{\mathrm{ct}}(1.16 \Omega)$ values compared to PEDOT $\left(\mathrm{ESR}=40.1 \Omega, R_{\mathrm{ct}}=3.7 \mathrm{k} \Omega\right), \operatorname{PrGO}\left(\mathrm{ESR}=39.3 \Omega, R_{\mathrm{ct}}=2.72 \Omega\right)$ and HKUST-1 $\left(\mathrm{ESR}=36.2 \Omega, R_{\mathrm{ct}}=2.56 \Omega\right)$. The lowest ESR value of PrGO/HKUST-1 reveals a good contact between the current collector and electrode material while the small $R_{\mathrm{ct}}$ shows a low resistance at the electrode/electrolyte interface, demonstrating high conductivity of PrGO/HKUST-1 44. Moreover, PrGO/HKUST-1 illustrates the shortest vertical line at low-frequency region, signifying a rapid ion diffusion rate ${ }^{45}$ within the electrode/electrolyte interface.

Inset Figure 6(b) displays an equivalent circuit that represents the electrochemical system of PrGO/HKUST-1 composite. The equivalent circuit consists of ESR, $R_{\mathrm{ct}}$, Warburg $(\mathrm{W})$ and the constant phase element (CPE). The double layer capacitor $\left(C_{\mathrm{dl}}\right)$ is replaced by CPE due to the electrode surface inhomogeneity ${ }^{7}$. Chi-square $\left(X^{2}\right)$ is the sum of the square differences between theoretical and experimental results ${ }^{46}$. From the fit and simulation analysis, the value of $x^{2}$ obtained is $7.2 \times 10^{-3}$, proving that the equivalent circuit is suitable for the electrochemical system of PrGO/HKUST-1.

The cycling stabilities of HKUST-1 and PrGO/HKUST-1 were evaluated over $4000 \mathrm{CV}$ cycles at $100 \mathrm{mV} / \mathrm{s}$. From Figure 6(c), the capacitance retention of PrGO/HKUST-1 is $95.5 \%$ compared to HKUST-1 (85.4\%), confirming excellent long-term stability of the PrGO/HKUST-1 device. A slight increment in capacitance retention (first 300 cycles) can be noticed in both HKUST-1 (121.3\%) and PrGO/HKUST-1 (103.2\%), indicating a self-activation process where electrolyte ions continuously penetrate all the active sites of the composite ${ }^{47}$. During the long-term cycling stability, HKUST-1 depicts an obvious decrease in specific capacitance compared to PrGO/HKUST-1 due to the swelling and shrinking properties of HKUST-1 during the redox reaction ${ }^{48}$. The high cycling stability of $\mathrm{PrGO} / \mathrm{HKUST}-1$ is due to the presence of $\mathrm{rGO}$, where it is able to provide high mechanical strength to the composite 49

(a)

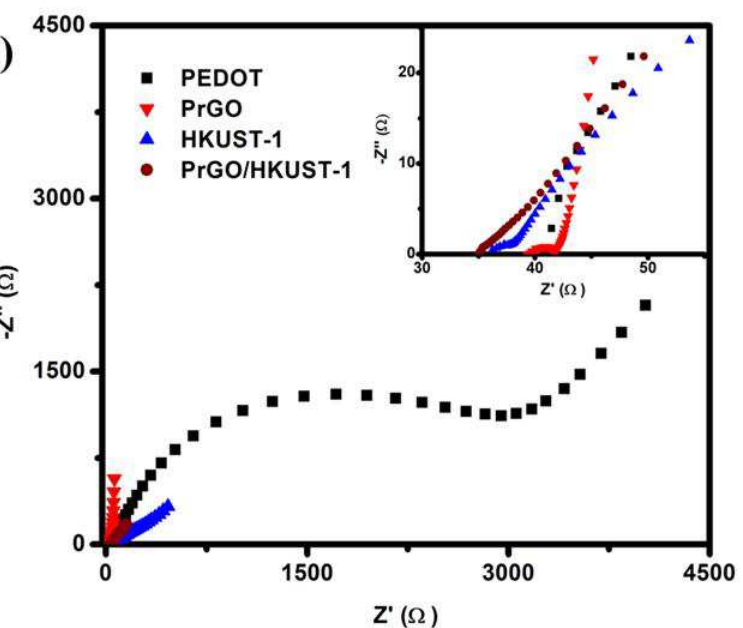

(b)

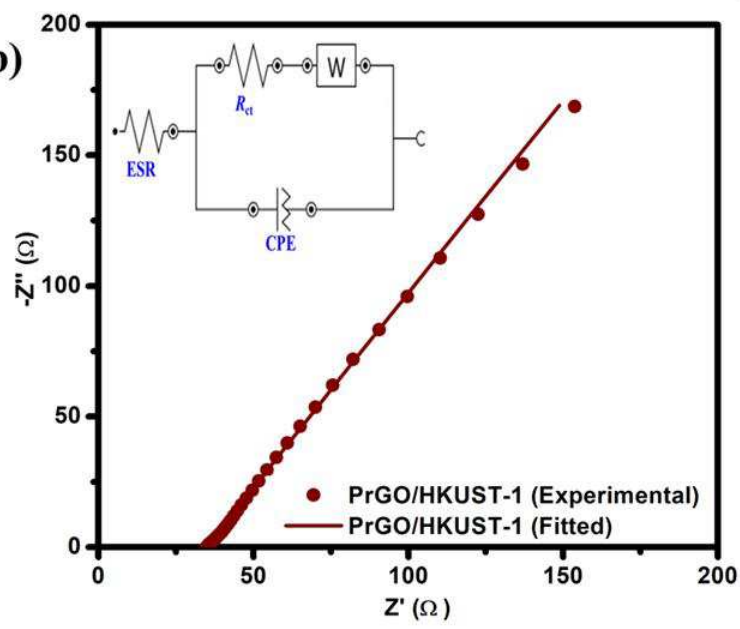

(c)

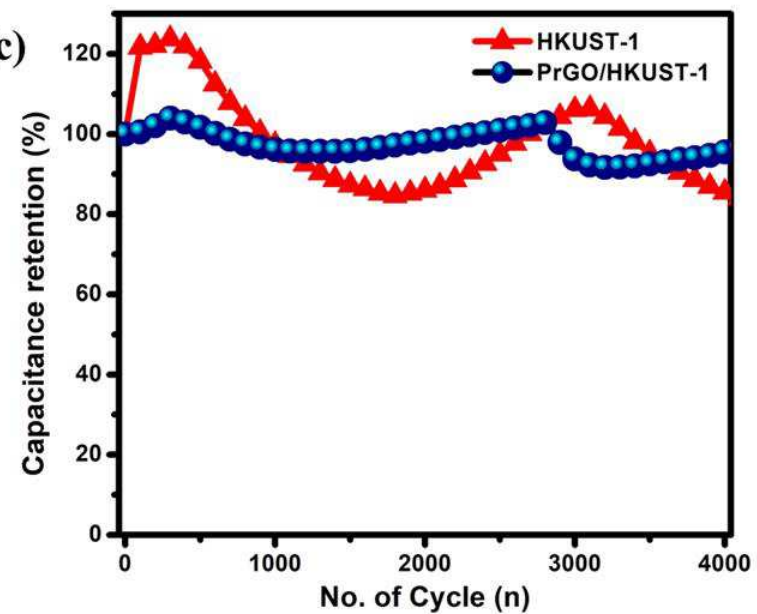


Figure 6: (a) Nyquist plots of PrGO, PEDOT, HKUST-1 and PrGO/HKUST-1 between $0.1 \mathrm{~Hz}$ and $10 \mathrm{kHz}$ (inset displays at high-frequency region). (b) Nyquist plot of PrGO/HKUST-1 with an equivalent circuit and (c) cycling stability over 4000 cycles at $100 \mathrm{mV} / \mathrm{s}$.

\section{Conclusions}

A novel PrGO/HKUST-1 composite was successfully synthesized as an outstanding supercapacitor device. The octahedral HKUST-1 on wrinkled-like sheet PrGO exhibited a unique morphology which boosts the electrochemical performance of the electrode by demonstrating a superior specific capacitance $(360.5 \mathrm{~F} / \mathrm{g})$, remarkable specific energy $(21 \mathrm{Wh} / \mathrm{kg})$ at a specific power of $479.7 \mathrm{~W} / \mathrm{kg}$ and excellent cyclability $(95.5 \%$ energy retention over 4000 cycles). Thus, the combination of PrGO and HKUST-1 with enhanced electrochemical performance is a promising energy storage material.

\section{Experimental}

Materials. Indium Tin Oxide (ITO) glasses (7 $\Omega$ ) was supplied by Xin Yan Technology Ltd. Acetone (99\%) and ethanol (95\%) were received from QRec and J.Kollin Chemical, respectively. GO was procured from Graphenea. Copper (II) nitrate trihydrate was acquired from Fisher Scientific while trimesic acid (BTC) was obtained from Sigma-Aldrich. Polyvinyl alcohol (PVA), N-methyl-2-pyrrolidone (NMP), 3,4-ethylenedioxythiophene (EDOT), carbon black, poly(vinylidene fluoride) (PVDF) were received from Sigma-Aldrich. Potassium chloride (KCl) was supplied by Fisher Scientific. Deionized (DI) water (18.5 M. $\mathrm{cm})$ from the Merck Millipore-Q was utilized in all experiments.

Preparation of PrGO/HKUST-1. PrGO was electrodeposited on ITO glass (current collector) at a fixed potential of $1.2 \mathrm{~V}$ for $10 \mathrm{~min}$ using an aqueous electrolyte consisting of $10 \mathrm{mM}$ EDOT along with $1 \mathrm{mg} / \mathrm{ml} \mathrm{GO}{ }^{7}$. For comparison, PEDOT was also prepared via a similar deposition technique using $10 \mathrm{mM}$ EDOT and $0.1 \mathrm{M} \mathrm{LiClO}_{4}$ in DI water. The electrodeposition of PEDOT and PrGO were carried out via potentiostat (Autolab PGSTAT204) in a three-electrode configuration where $\mathrm{Ag} / \mathrm{AgCl}$, ITO glass and platinum wire (Pt) were utilized as the reference electrode, working electrode and counter electrode, respectively.

Firstly, $0.88 \mathrm{~g}$ copper (II) nitrate trihydrate $\left(\mathrm{Cu}\left(\mathrm{NO}_{3}\right)_{2} .3 \mathrm{H}_{2} \mathrm{O}\right)$ was dissolved in $12 \mathrm{~mL}$ DI water, while $0.42 \mathrm{~g}$ trimesic acid (BTC) was dissolved in $12 \mathrm{~mL}$ ethanol. Next, the prepared mixtures were then mixed followed by stirring for 1 $\mathrm{hr}$. The resultant mixture was poured into a Teflon-lined autoclave, sealed and heated at $120^{\circ} \mathrm{C}$ for $16 \mathrm{hr}$. Finally, HKUST-1 was obtained and dried. A uniform HKUST-1 slurry was obtained by mixing HKUST-1, polyvinylidene fluoride (PVDF) (in N-methylpyrrolidone (NMP)), carbon black at 8:1:1 mass ratio ${ }^{45}$. HKUST-1 slurry (10 $\left.\mu \mathrm{L}\right)$ was drop coated on the PrGO layer $\left(1 \mathrm{~cm}^{2}\right)$ using a micropipette 50 to obtain PrGO/HKUST-1 composite. The PrGO/HKUST-1 was allowed to dry before proceeding with the electrochemical measurements.

Fabrication of symmetrical supercapacitor. A KCl/PVA gel was obtained by mixing $1 \mathrm{~g}$ PVA as well as $0.75 \mathrm{~g}$ $\mathrm{KCl}$ in $10 \mathrm{ml} \mathrm{DI}$ water, followed by vigorous stirring and heating $\left(\sim 90^{\circ} \mathrm{C}\right)$ until a clear and transparent gel was obtained ${ }^{51}$. Two identical PrGO/HKUST-1 electrodes $\left(1 \mathrm{~cm}^{2}\right)$ were then sandwiched together, separated by $\mathrm{KCl} / \mathrm{PVA}$ gel electrolyte. The KCl/PVA gel acted as an electrolyte and an ion-porous separator.

Material characterizations. The phase identification of composites was analyzed using X-Ray diffraction (XRD) (Shimadzu with $\mathrm{Cu}$ Ka radiation $(\lambda=1.54 \AA$ ). Raman spectroscopy (Alpha300 R microscopic confocal Raman spectrometer (WITec $\mathrm{GmbH}$ ), $532 \mathrm{~nm}$ ) was used to examine the functional groups of the as-prepared composites. The morphology of the composites was examined using field emission scanning microscopy (FESEM, JOEL JSMT600F).

Electrochemical analysis. The cyclic voltammetry (CV) and galvanostatic charge-discharge (GCD) analysis of individual electrodes were tested in $1 \mathrm{M} \mathrm{KCl}$ solution via a three-electrode configuration. The performance of the assembled devices was further tested in a two-electrode configuration utilizing CV, GCD, electrochemical impedance spectroscopy (EIS) as well as a cycling stability test. The CV analyses were performed at different scan rates $(5-100 \mathrm{mV} / \mathrm{s})$ whereas GCD analyses were recorded at different current densities $(1.0-2.0 \mathrm{~A} / \mathrm{g})$. EIS was conducted at a frequency range from $0.1 \mathrm{~Hz}$ to $10 \mathrm{kHz}$ and a perturbation amplitude of $5 \mathrm{mV}$ at an open circuit potential (OCP). The cycling stability test was measured over 4000 cycles. 


\section{References}

$1 \quad \mathrm{Fu}$, D. et al. Flexible solid-state supercapacitor fabricated by metal-organic framework/graphene oxide hybrid interconnected with PEDOT. Materials Chemistry and Physics 179, 166-173 (2016).

Zhu, M. et al. An electrochromic supercapacitor and its hybrid derivatives: quantifiably determining their electrical energy storage by an optical measurement. Journal of Materials Chemistry A 3, 21321-21327 (2015).

Dubal, D. P., Chodankar, N. R., Kim, D.-H. \& Gomez-Romero, P. Towards flexible solid-state supercapacitors for smart and wearable electronics. Chemical Society Reviews 47, 2065-2129 (2018).

4 Liu, Y.-P., Qi, X.-H., Li, L., Zhang, S.-H. \& Bi, T. MOF-derived PPy/carbon-coated copper sulfide ceramic nanocomposite as high-performance electrode for supercapacitor. Ceramics International (2019).

Samantara, A. K. \& Ratha, S. in Materials Development for Active/Passive Components of a Supercapacitor: Background, Present Status and Future Perspective 11-39 (Springer 2018). Abdah, M. A. A. M. et al. Potentiostatic deposition of poly (3, 4-ethylenedioxythiophene) and manganese oxide on porous functionalised carbon fibers as an advanced electrode for asymmetric supercapacitor. Journal of Power Sources 444, 227324 (2019).

Azman, N. H. N., Sulaiman, Y., Mamat, M. S., Nazir, M. \& Lim, H. N. Novel poly (3, 4ethylenedioxythiophene)/reduced graphene oxide incorporated with manganese oxide/iron oxide for supercapacitor device. Journal of Materials Science: Materials in Electronics 30, 1458-1467 (2019).

$\mathrm{Du}, \mathrm{W}$. et al. Advanced metal-organic frameworks (MOFs) and their derived electrode materials for supercapacitors. Journal of Power Sources 402, 281-295 (2018).

9 Zhao, Y. et al. Metal organic frameworks for energy storage and conversion. Energy storage materials 2, 35-62 (2016).

10 Li, Y. et al. MOF-Derived Metal Oxide Composites for Advanced Electrochemical Energy Storage. Small 14, 1704435 (2018).

11 Jafari, E. A., Moradi, M., Borhani, S., Bigdeli, H. \& Hajati, S. Fabrication of hybrid supercapacitor based on rod-like HKUST-1@ polyaniline as cathode and reduced graphene oxide as anode. Physica E: Low-dimensional Systems and Nanostructures 99, 16-23 (2018). Álvarez, J. R. et al. Structure stability of HKUST-1 towards water and ethanol and their effect on its $\mathrm{CO}_{2}$ capture properties. Dalton Transactions 46, 9192-9200 (2017).

13 Ramachandran, R., Zhao, C., Luo, D., Wang, K. \& Wang, F. Synthesis of copper benzene-1,3,5tricarboxylate metal organic frameworks with mixed phases as the electrode material for supercapacitor applications. Applied Surface Science 460, 33-39 (2018).

14 Van Ngo, T., Moussa, M., Tung, T. T., Coghlan, C. \& Losic, D. Hybridization of MOFs and graphene: A new strategy for the synthesis of porous 3D carbon composites for high performing supercapacitors. Electrochimica Acta 329, 135104 (2020).

15 Kulandaivalu, S. et al. A simple strategy to prepare a layer-by-layer assembled composite of $\mathrm{Ni}$-Co LDHs on polypyrrole/rGO for a high specific capacitance supercapacitor. RSC Advances 9, 40478-40486 (2019).

16 Zhu, J. et al. The effect of various electrolyte cations on electrochemical performance of polypyrrole/RGO based supercapacitors. Physical Chemistry Chemical Physics 17, 2866628673 (2015).

17 Wen, P. et al. Design and fabrication of carbonized rGO/CMOF-5 hybrids for supercapacitor applications. RSC Advances 6, 13264-13271 (2016). 

supercapacitor of reduced graphene oxide-metal organic framework composite coated on carbon fiber paper. Electrochimica Acta 157, 69-77 (2015).

19 Liu, Z. et al. Facile one-pot preparation of $\mathrm{Pd}-\mathrm{Au} / \mathrm{PEDOT} /$ graphene nanocomposites and their high electrochemical sensing performance for caffeic acid detection. RSC advances 6 , 89157-89166 (2016).

20 Li, D.-J. et al. Helical carbon tubes derived from epitaxial Cu-MOF coating on textile for enhanced supercapacitor performance. Dalton Transactions 47, 5558-5563 (2018).

21 Jiang, C., Chen, G. \& Wang, X. High-conversion synthesis of poly (3, 4ethylenedioxythiophene) by chemical oxidative polymerization. Synthetic metals 162,1968 1971 (2012).

22 Khalit, W. N. A. W., Mustafa, M. N. \& Sulaiman, Y. Synergistic effect of poly (3, 4ethylenedioxythiophene), reduced graphene oxide and aluminium oxide) as counter electrode in dye-sensitized solar cell. Results in Physics 13, 102355 (2019).

23 Zhang, Y., Liu, J., Zhang, Y., Liu, J. \& Duan, Y. Facile synthesis of hierarchical nanocomposites of aligned polyaniline nanorods on reduced graphene oxide nanosheets for microwave absorbing materials. RSC advances 7, 54031-54038 (2017).

24 Ahmed, S. \& Rafat, M. Hydrothermal synthesis of PEDOT/rGO composite for supercapacitor applications. Materials Research Express 5, 015507 (2018).

25 Jiang, L.-L. et al. Rapid electrochemical synthesis of HKUST-1 on indium tin oxide. RSC Advances 7, 9316-9320 (2017).

26 Raoof, J.-B., Hosseini, S. R., Ojani, R. \& Mandegarzad, S. MOF-derived Cu/nanoporous carbon composite and its application for electro-catalysis of hydrogen evolution reaction. Energy $\mathbf{9 0}$, 1075-1081 (2015).

27 Rebelo, S. L. et al. Progress in the Raman spectra analysis of covalently functionalized multiwalled carbon nanotubes: unraveling disorder in graphitic materials. Physical Chemistry Chemical Physics 18, 12784-12796 (2016).

28 Palaniselvam, T., Aiyappa, H. B. \& Kurungot, S. An efficient oxygen reduction electrocatalyst from graphene by simultaneously generating pores and nitrogen doped active sites. Journal of Materials Chemistry 22, 23799-23805 (2012).

29 Kuila, A., Maity, N., Layek, R. K. \& Nandi, A. K. On the pH sensitive optoelectronic properties of amphiphilic reduced graphene oxide via grafting of poly (dimethylaminoethyl methacrylate): a signature of $\mathrm{p}$-and n-type doping. Journal of Materials Chemistry A 2, 16039-16050 (2014).

30 Sofi, F. A., Bhat, M. A. \& Majid, K. $\mathrm{Cu}^{2+}-\mathrm{BTC}$ based metal-organic framework: a redox accessible and redox stable MOF for selective and sensitive electrochemical sensing of acetaminophen and dopamine. New Journal of Chemistry 43, 3119-3127 (2019).

31 Cen, B., Li, K., Lv, C. \& Yang, R. A novel asymmetric activated carbon electrode doped with metal-organic frameworks for high desalination performance. Journal of Solid State Electrochemistry, 1-11 (2020).

32 Zhang, S. et al. Polyaniline nanoparticle coated graphene oxide composite nanoflakes for bifunctional multicolor electrochromic and supercapacitor applications. Journal of Materials Science: Materials in Electronics, 1-12 (2019).

33 Zhou, K. et al. Polyaniline films with modified nanostructure for bifunctional flexible multicolor electrochromic and supercapacitor applications. Chemical Engineering Journal 345, 290-299 (2018).

34 Chen, G. Z. Understanding supercapacitors based on nano-hybrid materials with interfacial conjugation. Progress in Natural Science: Materials International 23, 245-255 (2013).

35 Zhao, D.-D., Xu, M. W., Zhou, W.-J., Zhang, J. \& Li, H. L. Preparation of ordered mesoporous nickel oxide film electrodes via lyotropic liquid crystal templated electrodeposition route. Electrochimica Acta 53, 2699-2705 (2008). 
36 Feng, K. et al. Study on the binder-free asymmetric supercapacitors with nano- $\mathrm{IrO}_{2}-\mathrm{ZnO} / \mathrm{Ti}$ as anode and $\mathrm{RuO}_{2}-\mathrm{MoO}_{3} / \mathrm{Ti}$ as cathode in $\mathrm{H}_{2} \mathrm{SO}_{4}$ electrolyte. Journal of Alloys and Compounds 819, 153385 (2020).

37 Guan, B. et al. Synthesis of hierarchical NiS microflowers for high performance asymmetric supercapacitor. Chemical Engineering Journal 308, 1165-1173 (2017).

38 Zhang, $\mathrm{C}$. et al. Hierarchically porous $\mathrm{Co}_{3} \mathrm{O}_{4} / \mathrm{C}$ nanowire arrays derived from a metal-organic framework for high performance supercapacitors and the oxygen evolution reaction. Journal of Materials Chemistry A 4, 16516-16523 (2016).

39 Abdah, M. A. A. M., Rahman, N. A. \& Sulaiman, Y. Ternary functionalised carbon nanofibers/polypyrrole/manganese oxide as high specific energy electrode for supercapacitor. Ceramics International 45, 8433-8439 (2019).

$40 \mathrm{Lim}, \mathrm{Y}$. et al. Potentiostatically deposited polypyrrole/graphene decorated nano-manganese oxide ternary film for supercapacitors. Ceramics International 40, 3855-3864 (2014).

41 Ravit, R., Abdullah, J., Ahmad, I. \& Sulaiman, Y. Electrochemical performance of poly (3, 4ethylenedioxythipohene)/nanocrystalline cellulose (PEDOT/NCC) film for supercapacitor. Carbohydrate polymers 203, 128-138 (2019).

42 Liu, M. et al. Core-shell reduced graphene oxide/ $\mathrm{MnO}_{x} @$ carbon hollow nanospheres for high performance supercapacitor electrodes. Chemical Engineering Journal 313, 518-526 (2017).

43 Zhao, X., Gnanaseelan, M., Jehnichen, D., Simon, F. \& Pionteck, J. Green and facile synthesis of polyaniline/tannic acid/rGO composites for supercapacitor purpose. Journal of Materials Science 54, 10809-10824 (2019).

44 Wang, L. et al. Hierarchical nanocomposites of polyaniline nanowire arrays on reduced graphene oxide sheets for supercapacitors. Scientific reports 3, 3568 (2013).

45 Guo, S. et al. (Metal-Organic Framework)-Polyaniline sandwich structure composites as novel hybrid electrode materials for high-performance supercapacitor. Journal of Power Sources 316, 176-182 (2016).

46 Chaudhari, S. \& Patil, P. Inhibition of nickel coated mild steel corrosion by electrosynthesized polyaniline coatings. Electrochimica acta 56, 3049-3059 (2011).

47 Ren, R. et al. Metallic CoS2 nanowire electrodes for high cycling performance supercapacitors. Nanotechnology 26, 494001 (2015).

48 Aljammal, N., Jabbour, C., Chaemchuen, S., Juzsakova, T. \& Verpoort, F. Flexibility in MetalOrganic Frameworks: A Basic Understanding. Catalysts 9, 512 (2019).

49 Zhang, J. \& Zhao, X. Conducting polymers directly coated on reduced graphene oxide sheets as high-performance supercapacitor electrodes. The Journal of Physical Chemistry C 116, 5420-5426 (2012).

50 Ojha, G. P., Pant, B., Park, S.-J., Park, M. \& Kim, H.-Y. Synthesis and characterization of reduced graphene oxide decorated with $\mathrm{CeO}_{2}$-doped $\mathrm{MnO}_{2}$ nanorods for supercapacitor applications. Journal of Colloid and Interface Science 494, 338-344 (2017).

51 Chen, Q. et al. Effect of different gel electrolytes on graphene-based solid-state supercapacitors. RSC Advances 4, 36253-36256 (2014).

\section{Acknowledgement}

The authors sincerely thank the Universiti Putra Malaysia Grant (GP-IPS/2017/9580500) for their financial assistance.

\section{Author contributions}


Y.S conceived the idea and supervised all the experiments. D.M carried out all the experiments, analyzed the data and wrote the main manuscript. M.A.A.M.A, N.H.N.A, T.B.S.A.R and Y.S reviewed and approved the manuscript.

\section{Competing interests}

The authors declare no competing interests. 


\section{Figures}

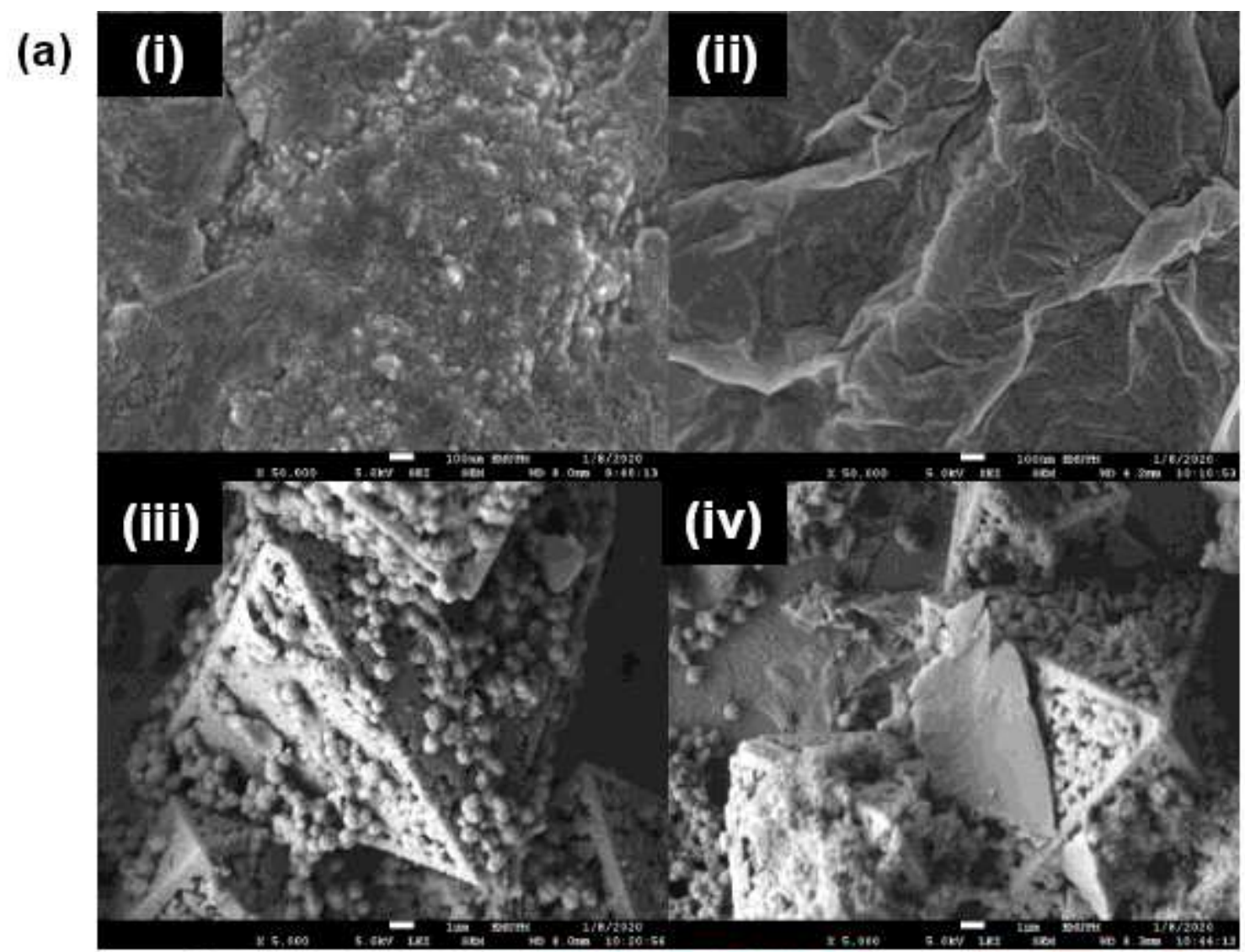

(b)
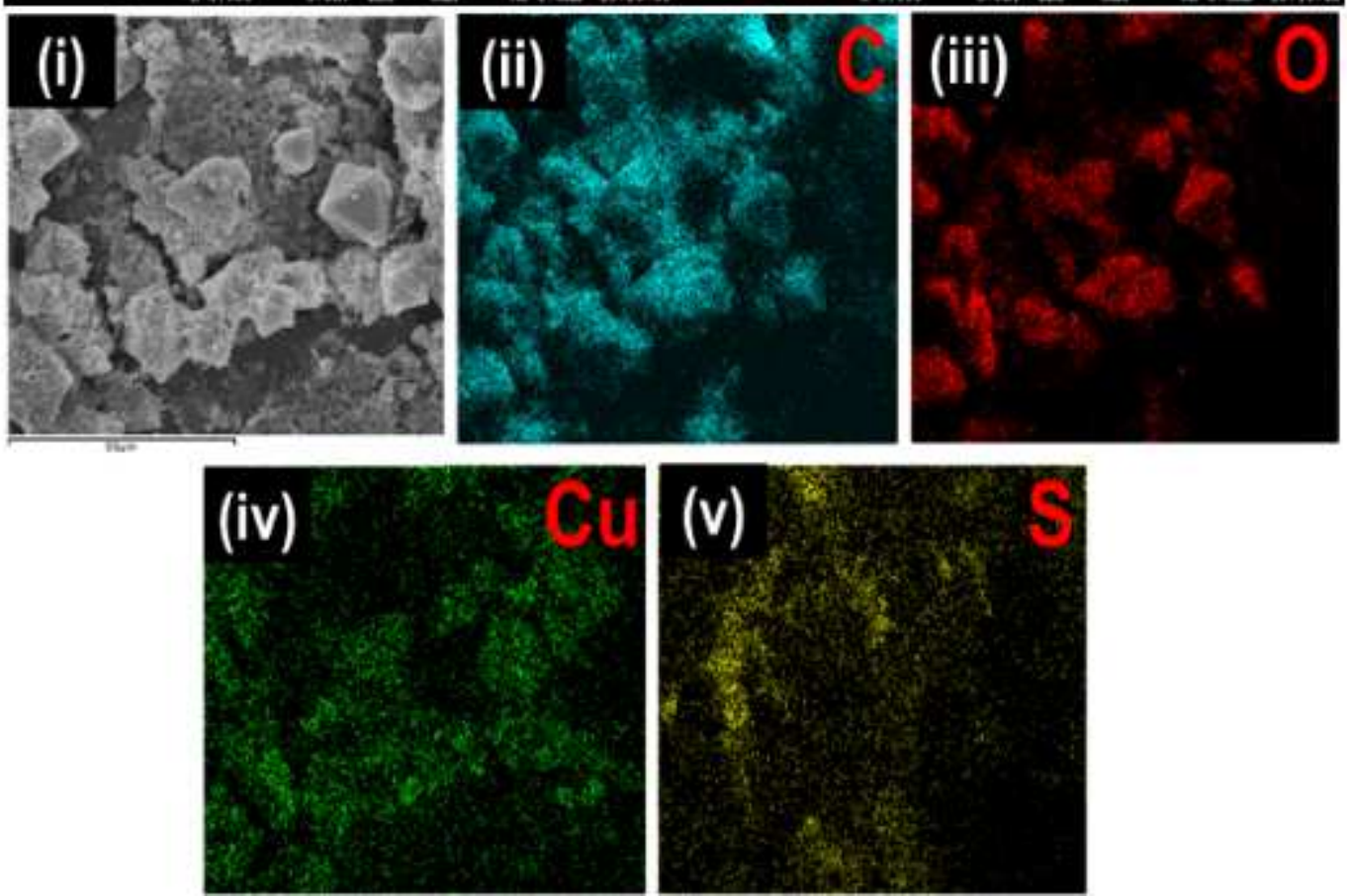

Figure 1

(a) FESEM images of (i) PEDOT (scale bar: $100 \mathrm{~nm}$ ), (ii) PrGO (scale bar: $100 \mathrm{~nm}$ ), (iii) HKUST-1 (scale bar: $1 \mu \mathrm{m}$ ), (iv) PrGO/HKUST-1 (scale bar: $1 \mu \mathrm{m}$ ) and (b) elemental mapping of (i) PrGO/HKUST-1 (scale bar: $50 \mu \mathrm{m}$ ) with (ii) $\mathrm{C}$, (iii) $\mathrm{O}$, (iv) $\mathrm{Cu}$ and (v) S distributions. 


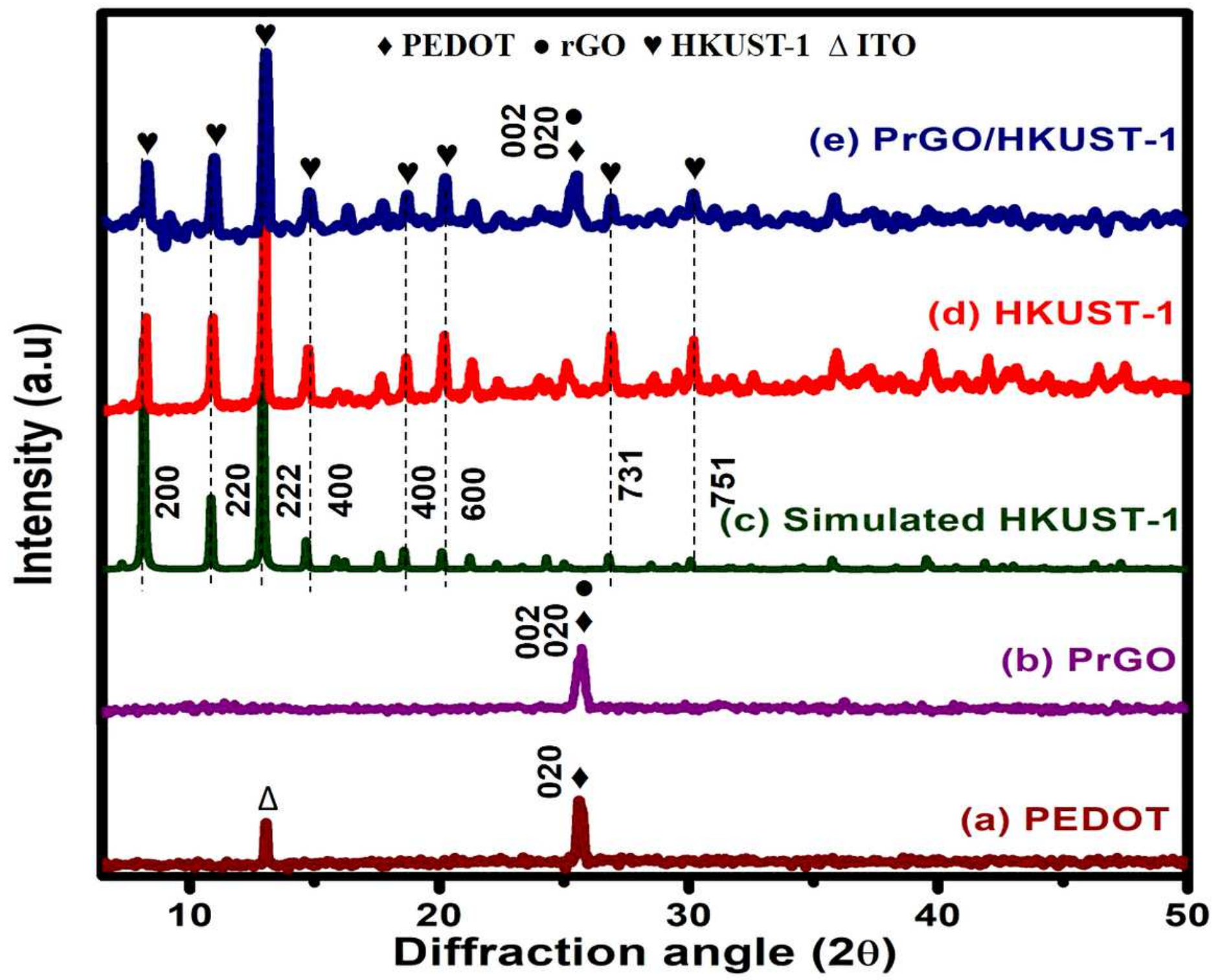

Figure 2

XRD patterns of (a) PEDOT, (b) PrGO, (c) simulated HKUST-1, (d) HKUST-1 and (e )PrGO/HKUST-1. 


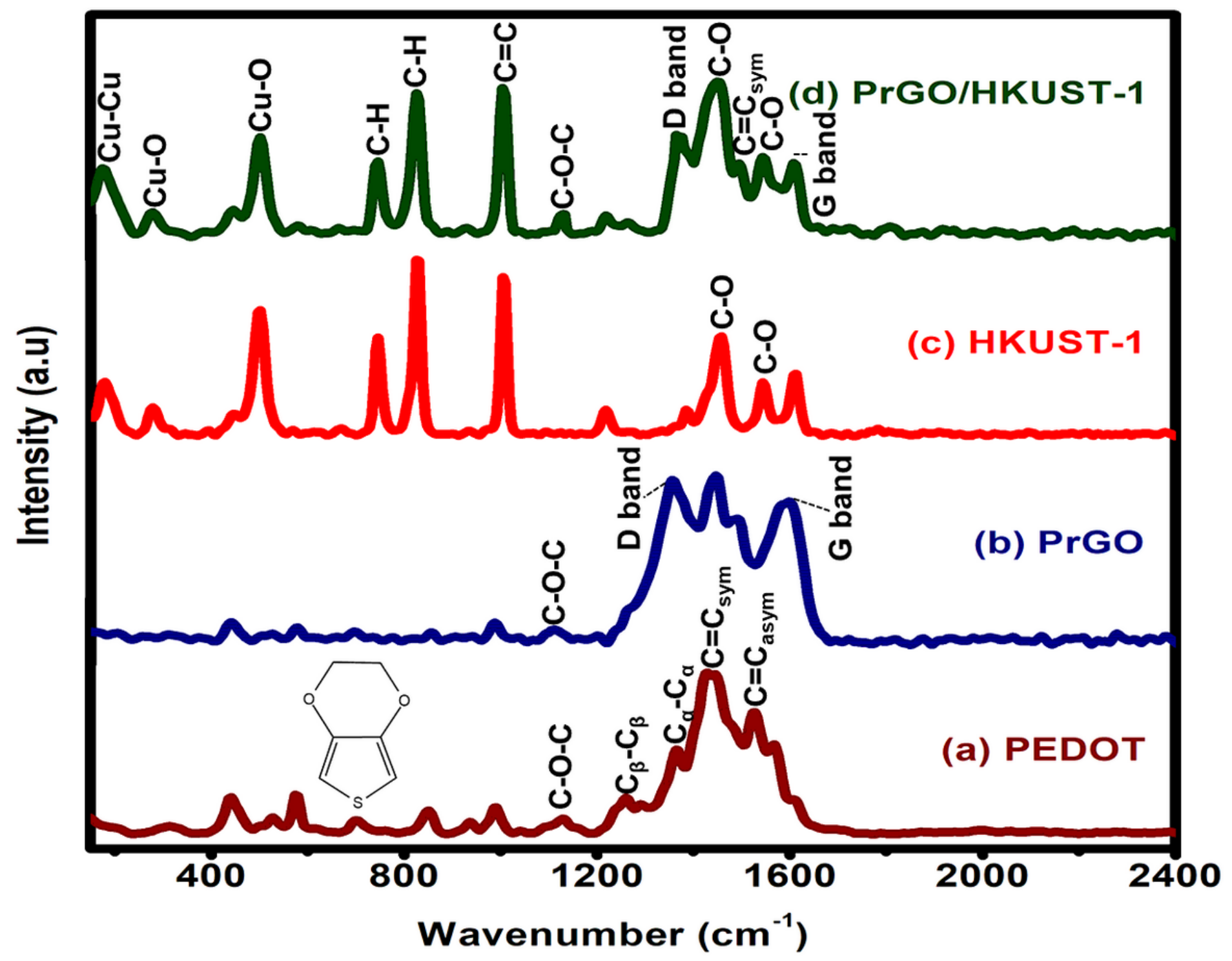

Figure 3

Raman spectra of (a) PEDOT, (b) PrGO, (c) HKUST-1 and (d) PrGO/HKUST-1 

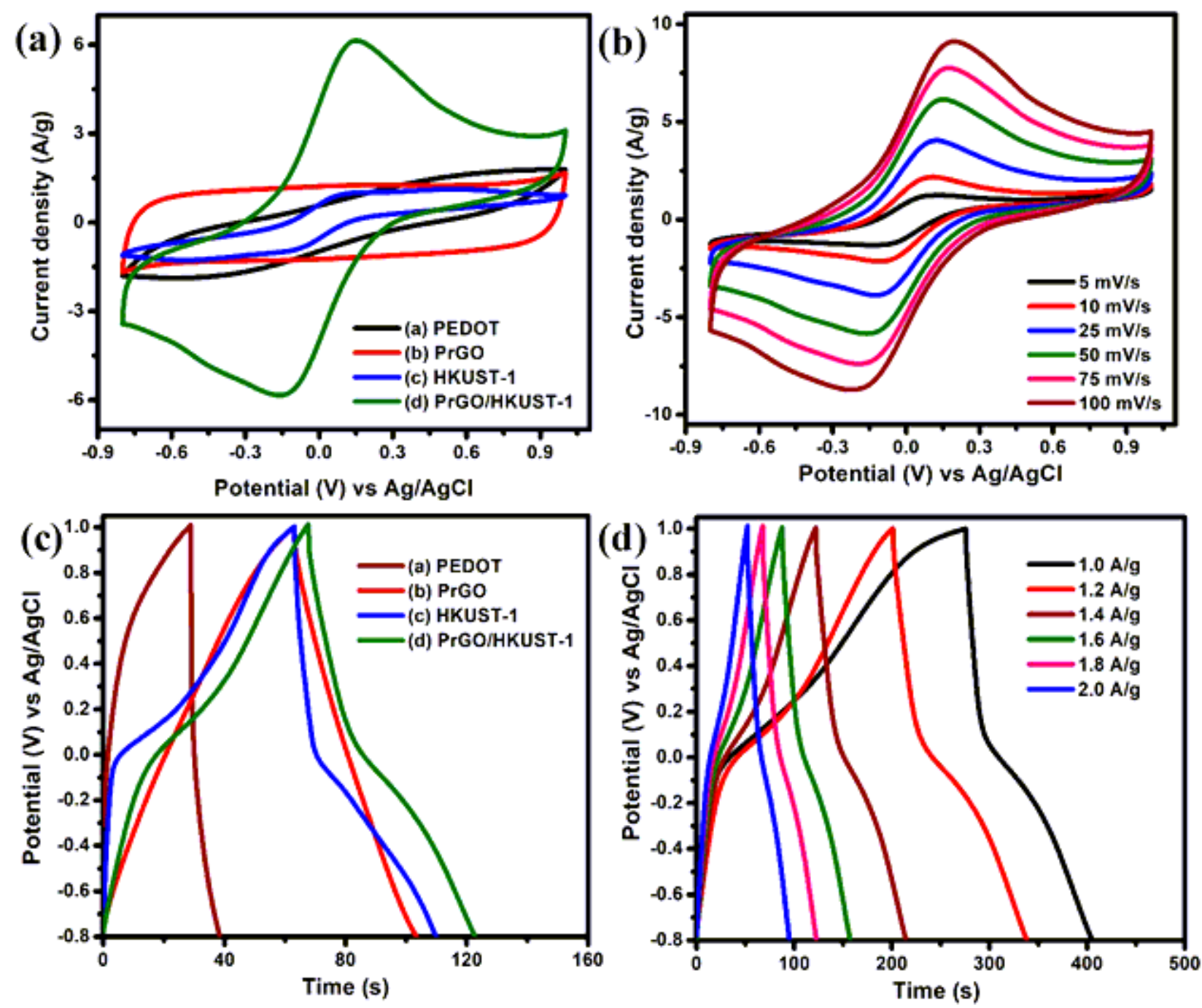

Figure 4

(a) CV curves of PEDOT, PrGO, HKUST-1 and PrGO/HKUST-1 at $50 \mathrm{mV} / \mathrm{s}$, (b) CV curves of PrGO/HKUST-1 at various scan rates (5-100 mV/s), (c) GCD analysis of PEDOT, PrGO, HKUST-1, PrGO/HKUST-1 and (d) GCD plots of PrGO/HKUST-1 at various current density (1.0-2.0 A/g). 

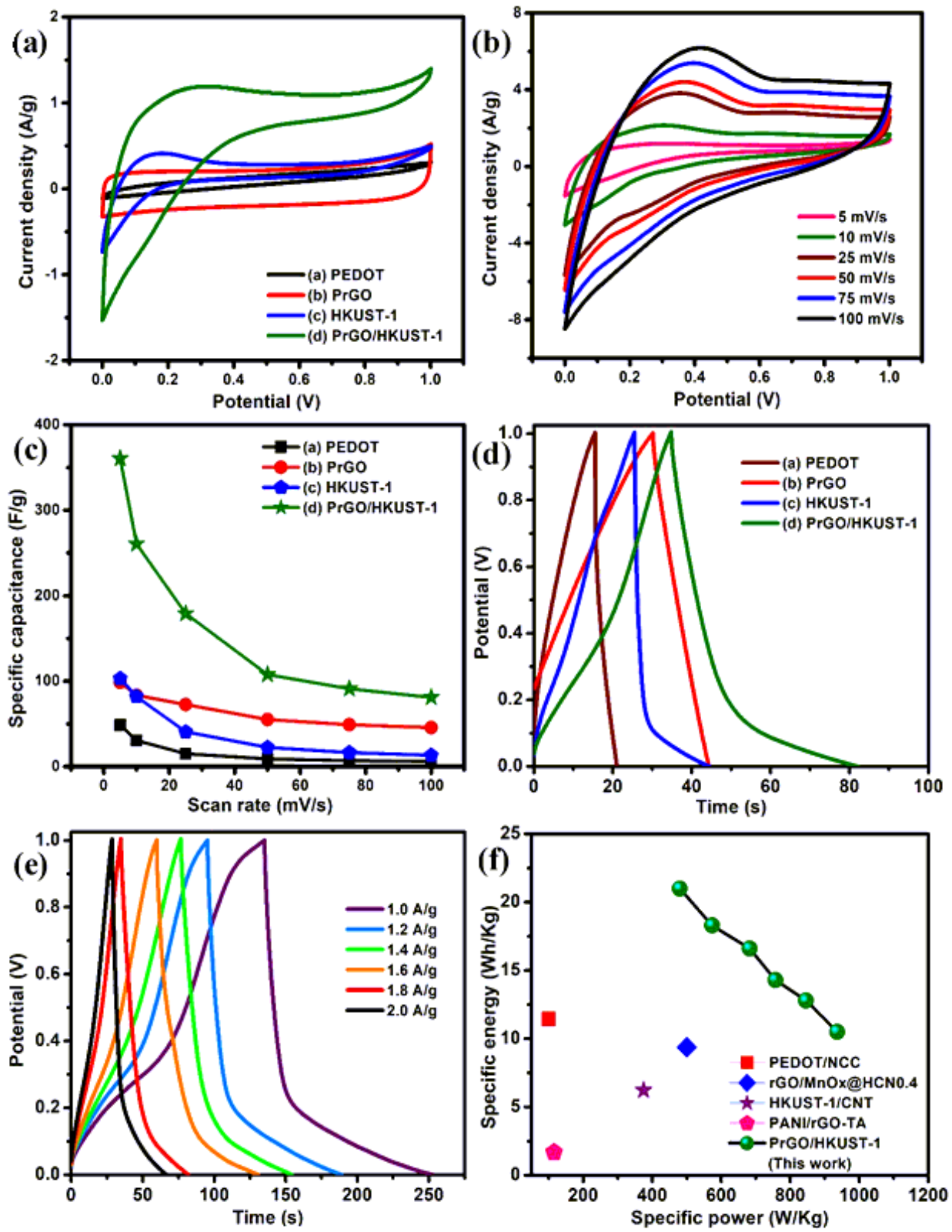

Figure 5

(a) CVs of PrGO, PEDOT, HKUST-1 and PrGO/HKUST-1 using KCl/PVA gel electrolyte at $5 \mathrm{mV} / \mathrm{s}$, (b) CVs of symmetrical PrGO/HKUST-1 at different scan rates (5- $100 \mathrm{mV} / \mathrm{s}$ ) and (c) graph of specific capacitance versus scan rate for PrGO, PEDOT, HKUST-1 and PrGO/HKUST-1, (d) GCD analysis of PEDOT, PrGO, HKUST-1 and PrGO/HKUST-1 at a current density of $1.8 \mathrm{~A} / \mathrm{g}$ using KCl/PVA gel electrolyte, (e) GCD 
analysis of symmetrical PrGO/HKUST-1 at various current densities (1.0-2.0 A/g), (f) Ragone plots of PrGO/HKUST-1.
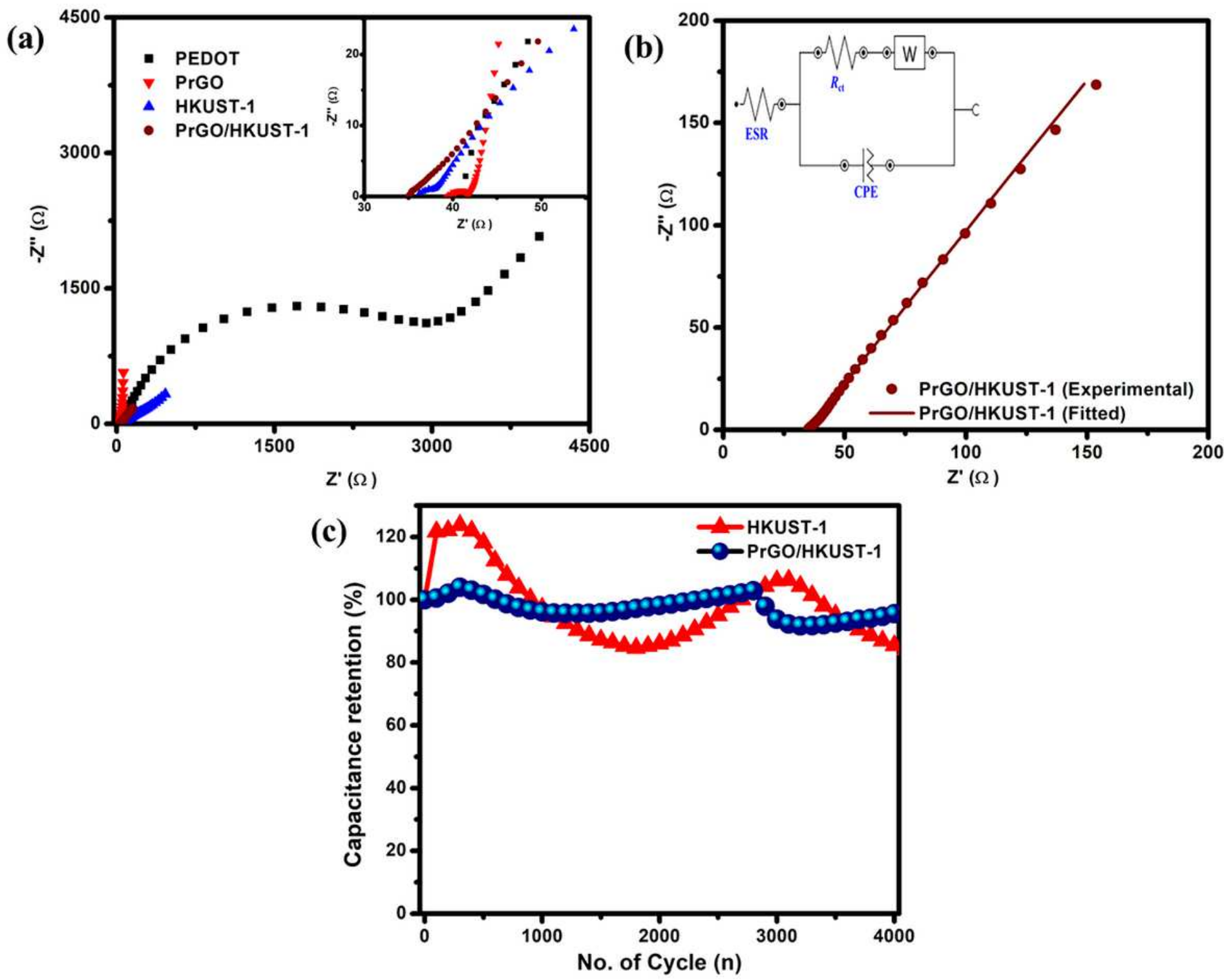

Figure 6

(a) Nyquist plots of PrGO, PEDOT, HKUST-1 and PrGO/HKUST-1 between $0.1 \mathrm{~Hz}$ and $10 \mathrm{kHz}$ (inset displays at high-frequency region). (b) Nyquist plot of PrGO/HKUST-1 with an equivalent circuit and (c) cycling stability over 4000 cycles at $100 \mathrm{mV} / \mathrm{s}$. 